\title{
IMFEDK
}

\section{Evolution of Low-Voltage CMOS Digital VLSI Circuits Using Bootstrap Technique}

\author{
James B. Kuo \\ Dept of Electrical Engineering, Rm 338 \\ National Taiwan University \\ Taipei, Taiwan 106-17 \\ Email: j.kuo@ieee.org
}

\begin{abstract}
This paper reports the evolution of the low-voltage CMOS digital VLSI circuits using bootstrap technique. Combining bootstrap and DTMOS techniques, low-voltage CMOS digital circuits using a power supply as low as $0.5 \mathrm{~V}$ are feasible for low-power VLSI system applications.
\end{abstract}

\section{INTRODUCTION}

For next-generation CMOS VLSI circuits, low supply voltage is the trend [1] [2]. Since the threshold voltage of the CMOS devices cannot be scaled down accordingly with the supply voltage, designing a CMOS digital circuit using a low supply voltage for the next-generation CMOS VLSI is a challenge. In this paper, the evolution of the low-voltage CMOS digital VLSI circuits using bootstrap technique is described. In the following sections, the bootstrap technique is presented first, followed by bootstrapped dynamic and static circuits.

\section{Bootstrap Technique}

Fig. 1 shows the bootstrap technique used in a CMOS large-load driver circuit [1]. Capacitor $\mathrm{C}_{\mathrm{bp}}$ and NMOS devices $\mathrm{M}_{\mathrm{N} 1 \mathrm{~b}} / \mathrm{M}_{\mathrm{N} 2 \mathrm{~b}}$ are used for the bootstrapped pull-up operation and capacitor $\mathrm{C}_{\mathrm{bn}}$ and PMOS devices $M_{P_{1 b}} / M_{P 2 b}$ are used for the bootstrapped pull-down operation. Fig. 2 shows the equivalent circuit of this bootstrapped CMOS large-load driver circuit during the pull-up transient [3]. Prior to the ramp-up period, $\mathrm{C}_{b p}$ has been charged to $\mathrm{V}_{\mathrm{DD}}$. Due to the charge stored in $C_{b p}$, after the ramp, $M_{p 1}$ has been driven by a minus gate voltage, hence a faster pull-up-bootstrap technique. As described in the following sections, the bootstrap technique has been used in the dynamic and static circuits to enhance the speed performance.

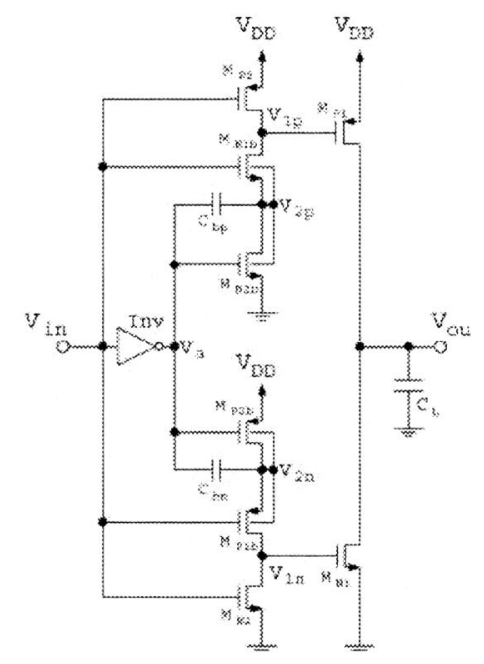

Fig. 1. Bootstrap technique used in a CMOS large-load driver circuit [3].

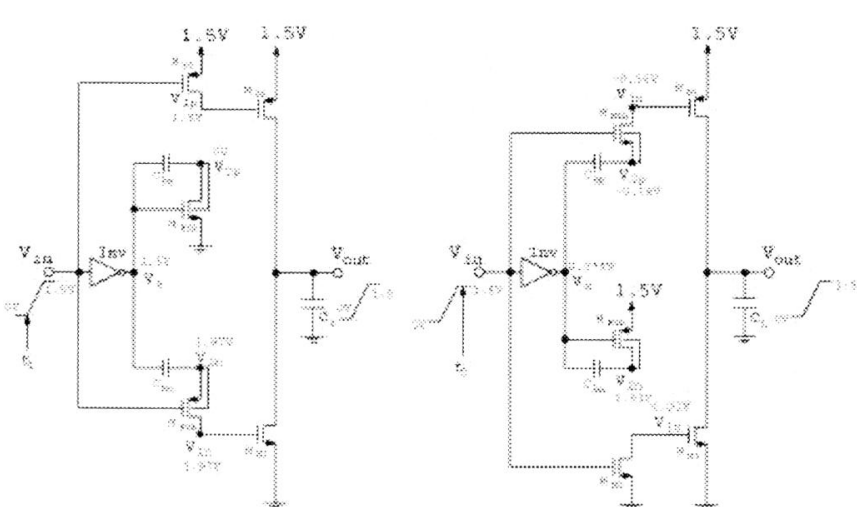

Fig. 2. Equivalent circuit of the bootstrapped CMOS large-load driver circuit during the pull-up transient [3].

\section{Bootstrapped Dynamic Logic CiRcuits}

Fig. 3 shows the bootstrapped dynamic logic (BDL) circuit, which is composed of the dynamic logic circuit and the bootstrapper circuit. During the precharge period (CK low), the bootstrap capacitor $C_{b}$ is charged to $V_{D D}$. During the logic evaluation period (CK high), if both inputs are high, owing to the charge in the bootstrap capacitor, $V_{b}$ is bootsrapped to over $V_{D D}$, hence the driving capability of the output is enhanced. 


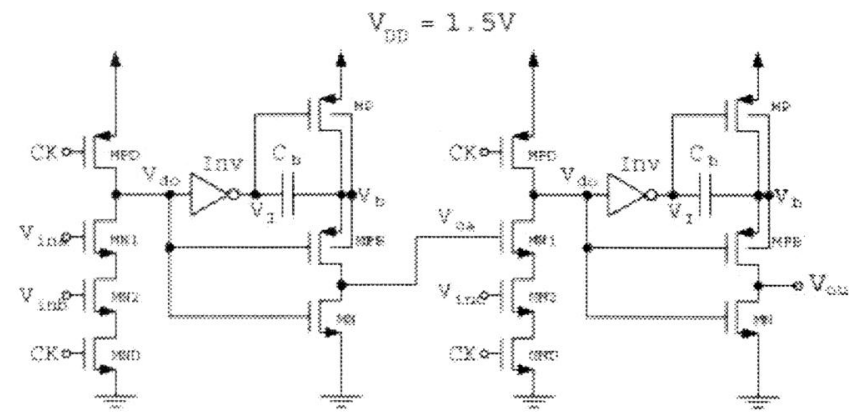

T - tyoe BDs

Fig. 3 CMOS bootstrapped dynamic logic (BDL) 2-input AND circuit [4].

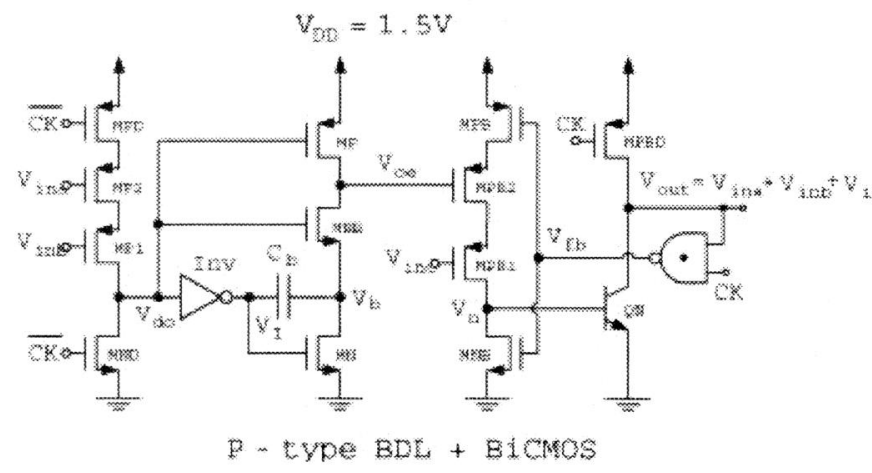

Fig. 4. 1.5V BiCMOS dynamic logic (BDL) circuit with the bootstrap technique [4].

Using the bootstrapper circuit, the speed performance of a $1.5 \mathrm{~V}$ BiCMOS boostrapped dynamic logic (BiCMOS BDL) as shown in Fig. 4 can be improved [4]. As shown in the figure, in this BiCMOS BDL circuit, it has a BiPMOS pull-down dynamic logic circuit and a p-type BDL circuit with a p-type bootstrapper circuit, which is complementary to the one as shown in Fig. 3.

Fig. 5 shows a three-input NAND circuit using the 1.5V CMOS all-N-logic TSP BDL circuit [5]. As shown in the figure, it is composed of an $\mathrm{n} 1$ block, which is identical to the n-type BDL as shown in Fig. 3, and an n2 block, which is derived from a p-type BDL circuit as shown in Fig. 4 to avoid using slower PMOS devices.

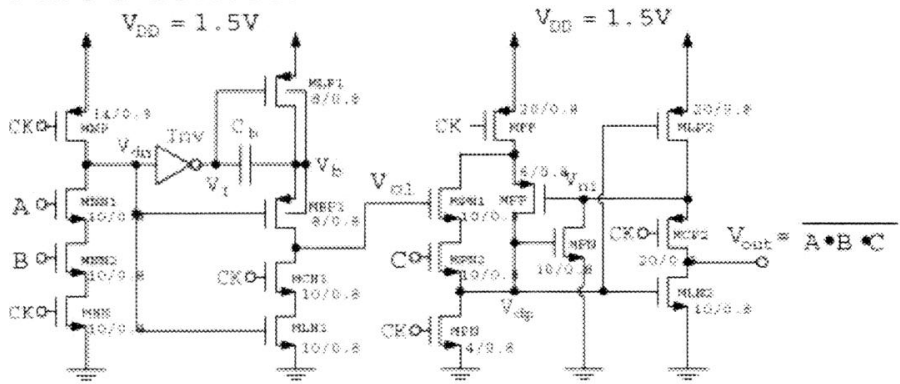

Fig. 5. Three-input NAND circuit using the 1.5V CMOS all-N-logic TSP BDL circuit [5].
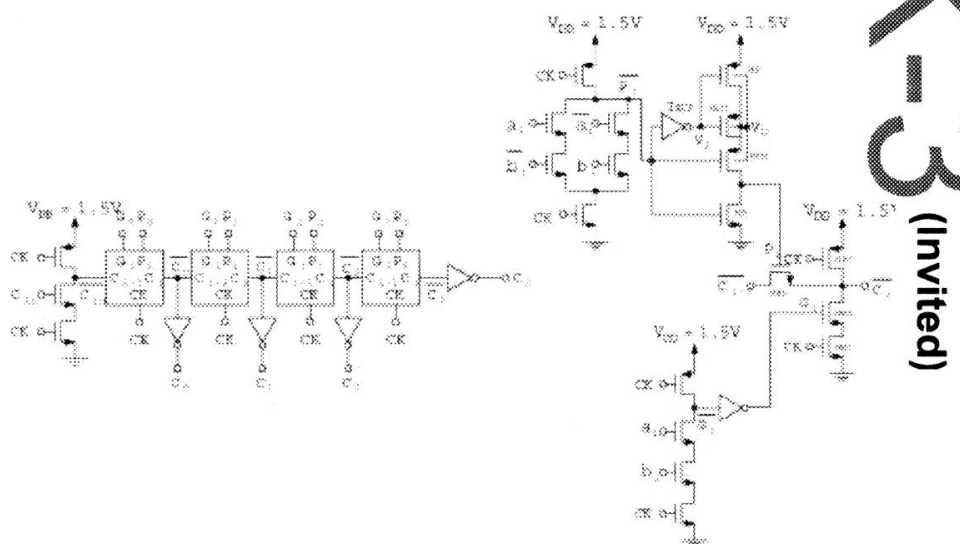

Fig. 6. 1.5V CMOS bootstrapped PT-based carry look-ahead circuit [6].

Fig. 6 shows the $1.5 \mathrm{~V}$ CMOS bootstrapped pass transistor (PT)-based carry look-ahead (CLA) circuit [6]. As shown in the figure, the bootstrapper circuit, which functions as an inverter with its output high level boosted to over $V_{D D}$, is used to boost the input signal to the gate of the pass transistors used in producing the propagate signal. With the gate overdrive voltage, the pass transistor can turn on earlier and have a larger current driving capability. Owing to the critical path formed by the serial-connected pass transistors, which are controlled by the propagate signals, the voltage overshoot at the output of the bootstrapper circuit enhances the speed performance of this PT-based Manchester carry chain circuit. Therefore, the bootstrapper circuit is especially advantages for low supply voltage applications.

\section{Bootstrapped Static Circuits}

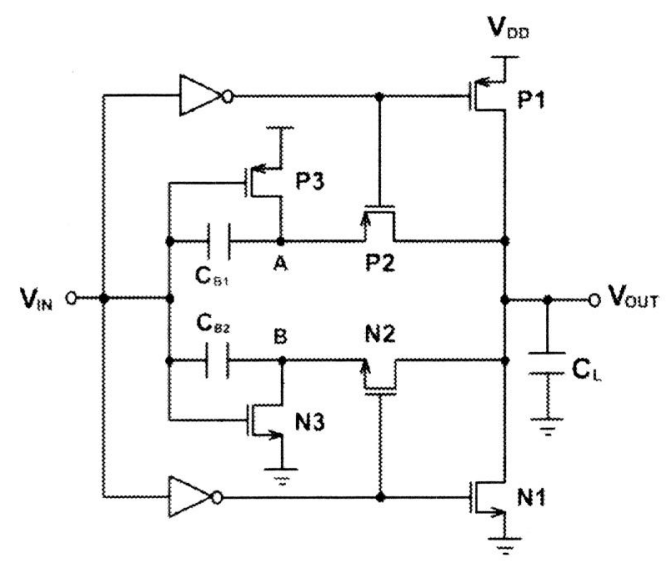

Fig. 7. Sub-1V CMOS load driver circuit using the direct bootstrap technique [7].

In addition to dynamic circuits, bootstrap technique has been used in the static circuits to enhance the speed performance. In this section, the direct bootstrapped circuit, the $0.5 \mathrm{~V}$ bootstrapped SOI CMOS load driver 
and the bootstrapped adiabatic circuit for lowpower systems are described.

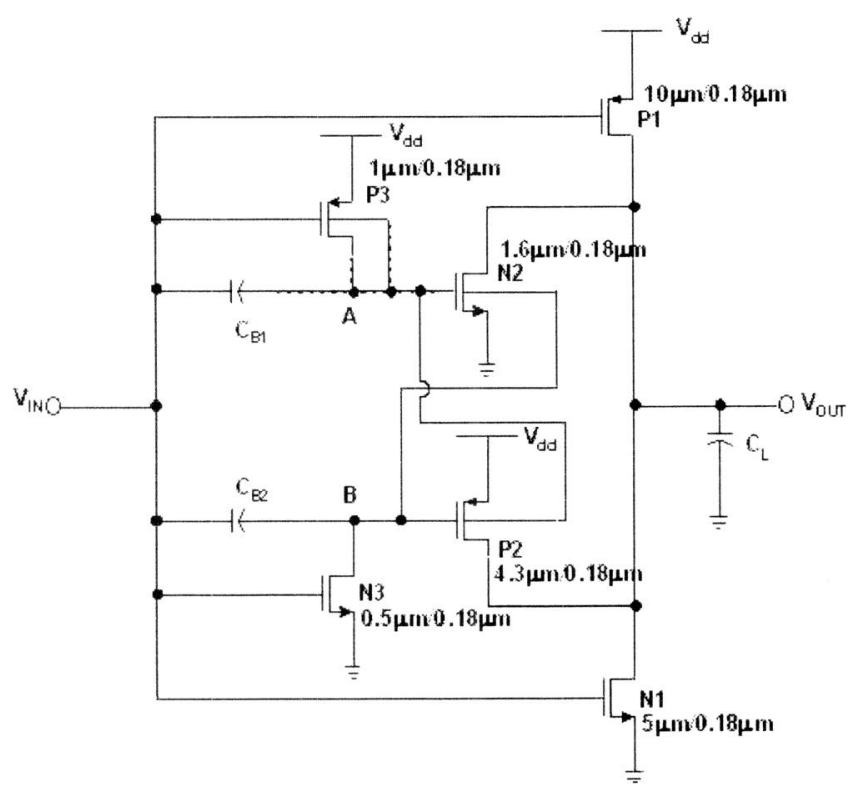

Fig. $80.5 \mathrm{~V}$ SOI CMOS inverting driver circuit using DTMOS/bootstrap technique [9].

The bootstrap technique described in Fig. 1 is called indirect bootstrap technique since it is applied at the gate of the output devices in a driver circuit, which may not be effective in shortening the switching speed of the output. Fig. 7 shows a sub-1V CMOS load driver circuit using the direct bootstrap technique [7]. As shown in the figure, in this direct bootstrap technique, the bootstrap capacitor $\mathrm{C}_{\mathrm{B} 1} / \mathrm{C}_{\mathrm{B} 2}$ is connected to the output node via $\mathrm{P} 2 / \mathrm{N} 2$, instead of via the gate of the output device as in other indirect bootstrap technique. Owing to the charge stored in the bootstrap capacitor $\mathrm{C}_{\mathrm{B} 1} / \mathrm{C}_{\mathrm{B} 2}$ prior to the pull-up/pull-down transient, the output can be pulled up/down quickly.

Combining with DTMOS technique [8], the bootstrap technique has been used in the SOI CMOS load driver using a very low power supply voltage. Fig. 8 shows a $0.5 \mathrm{~V}$ CMOS inverting driver using DTMOS/bootstrap technique [9]. As shown in the figure, this driver is composed of two bootstrap capacitors with the DTMOS technique for magnifying the input signals to overcome the shortage of the gate voltage over-drive problems for ultra lowvoltage operation such as $0.5 \mathrm{~V}$, which is comparable to or smaller than the magnitude of the threshold voltage of the devices used. The bootstrap capacitor $\mathrm{C}_{\mathrm{B} 1} / \mathrm{C}_{\mathrm{B} 2}$ with the precharge device $\mathrm{P} 3 / \mathrm{N} 3$ and the driver device N2/P2 with its body controlled by the DTMOS technique has been used to resolve the problem associated with the shortage of gate voltage over-drive.
CMOS adiabatic logic circuits using differential switch logic (DSL) are effective in achieving goals in low power consumption [1].

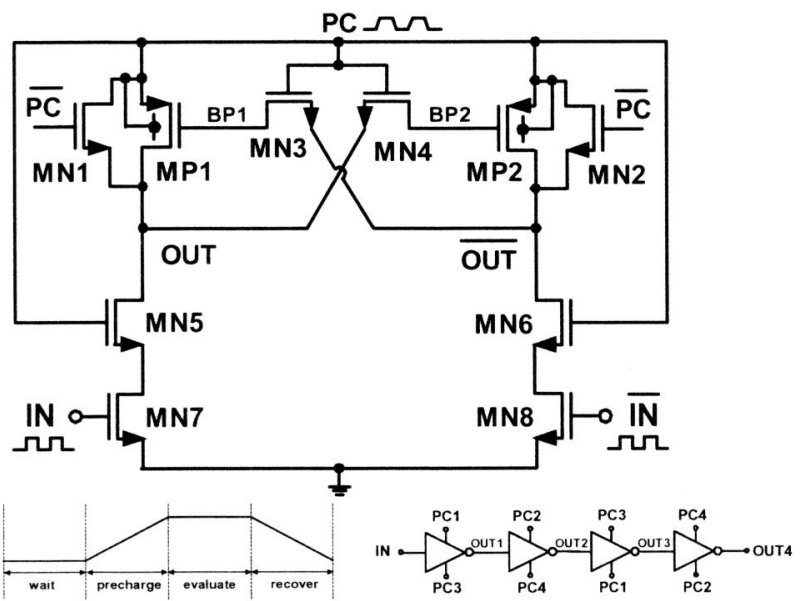

Fig. $90.8 \mathrm{~V}$ CMOS adiabatic differential switch logic (ADSL) circuit using the bootstrap technique [10].

Fig. 9 shows a $0.8 \mathrm{~V}$ CMOS adiabatic differential switch logic (ADSL) circuit using the bootstrap technique [10]. As shown in the figure, this ADSL circuit is derived from a DSL circuit with the cross-coupled bootstrap devices MN3/MN4 to enhance its switching performance. Owing to the capacitance coupling of the gate-drain capacitance of MN3/MN4, the voltage of the internal node BP1/BP2 could be bootstrapped. Thus the flow of charge stored at the output node is enhanced and hence the speed performance is raised.

\section{REFERENCES}

[1] J.Kuo, "LV CMOS VLSI Ckts," Wiley, NY 99. [2] J.Kuo,"LV SOI CMOS VLSI Dev/Ckts," Wiley, NY, 01.

[3] J.Lou \& J.Kuo, "A 1.5V FS Bt CMOS Lg Cap-Ld Dr," IEEE JSSC,119-21, 97.

[4] J.H. Lou \& J.B. Kuo, "1.5V CMOS and BiCMOS BDL," S.VLSI TSA, 279-82, 97.

[5] J.Lou \& J.Kuo, "1.5V CMOS All-N-Log TSP Bt BDL for LV Op," IEEE TCAS, 628-31, 99.

[6] J.Lou \& J.Kuo, "A 1.5-V Bt PT-B Man Carry C for CLA Add," IEEE TCAS, 1191-4, 98.

[7] P.Chen \& J.Kuo,"Sub-1V CMOS Dr Using DB Tech," Elec.Lett. 265-6, 02.

[8] F.Assaderaghi et al, "DTMOS for UltraLow-Volt VLSI," IEEE TED, 414-422, 1997.

[9] J.Chen \& J.Kuo, "ULV SOI CMOS Dr Based on Bt Tech," Elec. Lett. 183-5, 03.

[10] Y.Zhang,H.Chen \& J.Kuo, "0.8V CMOS Adiabatic Diff Sw Logic Using Bt Tech for LV LP VLSI," Elec. Lett., 1497-9, 02. 